\title{
General and particular aspects regarding conceiving and manufacturing fixed implant-supported prosthetic restorations
}

\author{
Sorin Nicolae Popescu', Mirel Toma', Florentina Radu², Gabriel Ciochinda', \\ Cristina Nicoleta Mihai', Viorel Stefan Perieanu', Ileana Ionescu', \\ Madalina Violeta Perieanu', Camelia Ionescu', Irina Adriana Beuran', \\ Gabriela Iorgulescu' ${ }^{1}$, Oana-Cella Andrei ${ }^{1}$, Liliana Burlibasa ${ }^{3}$, \\ Corina Marilena Cristache ${ }^{1}$, Mihai David ${ }^{1}$, Luminita Oancea ${ }^{1}$, Mihai Burlibasa ${ }^{1}$ \\ 1"Carol Davila" University of Medicine and Pharmacy, Bucharest, Romania \\ ${ }^{2}$ Private dental practice, Bucharest, Romania \\ ${ }^{3}$ Faculty of Biology, University of Bucharest, Romania
}

\begin{abstract}
Introduction. In general, implant-prosthetic restorations, whether they are fixed or mobile, and regardless of their location (maxillary or mandibular), imply slightly different design and conceiving criteria, compared to the conventional prosthetic restorations already known.

Purpose. In this material we tried to present some general and particular aspects regarding the design and manufacture of fixed implant-prosthetic restorations.

Material and method. The study took place between September and December 2019 with the help of 31 dentists from Bucharest.

Results and discussions. Following the application of the questionnaire, we were able to outline some aspects, frequently encountered in practice, by dentists who perform implant-prosthetic restorations related to occlusal balancing and the forces that appear at the level of implant restorations.

Conclusions. The correct analysis and evaluation of the occlusal contacts and forces, leads to an increase in the life expectancy of prosthetic work as well as of their support (dental or implant).
\end{abstract}

Keywords: fixed implant-prosthetic restorations, dental implants, dental occlusion

\section{INTRODUCTION}

As it is well known, it is mandatory that the dento-maxillary system prosthetically rehabilitated with the help of dental implants has a dynamic, morphological and functional balance, both at the level of each component and between its components. Thus, if we talk about the "classic", we can argue that partial edentations are restored in most cases either with the help of multidimensional fixed prosthetic restorations (dental bridges) or through partial dentures. Fixed prosthetic restorations on multiple teeth are maintained by cementing on previously natural teeth shaped with dental drills, while partial dentures are maintained with the help of cast clasps and/or special systems (1-8).

But due to a truly remarkable technological evolution in the field of oral implantology, in addition to the temporary dentition and the definitive dentition already known, the insertion of dental implants and creation of prosthetic restoration on them led to the appearance of a so-called "third dentition", because the functions of the dento-maxillary system (mastication, phonation and aesthetics) are accom- 
plished through dental implants, and now they are used to support bridges, crowns, partial and total dentures (1-8).

\section{PURPOSE}

In partial edentation, the remaining teeth are important prosthetic elements, and the choice and positioning of future dental implants will be made according to their status. The teeth implantation axis is different, and rarely two teeth are implanted the same way and never perpendicular on the occlusal plane or on the bone base. The roots of the maxillary teeth are convergent to the apex, and those of the mandibular teeth are divergent to the apex, which explains the lack of parallelism between the teeth. The forces exerted during the functional movements of the mandible tend to mobilize the teeth in bone, and they oppose to this phenomenon through periodontal ligaments (1-8).

But, when it is desired to treat an edentation with implant-supported prosthetic restoration, it is necessary to take into account the implantation of each tooth present on the arch, but also the implantation that the remaining teeth had, because the bone-implant interface must be at least equal to that of the tooth being replaced. When certain forces are exerted on a tooth, the periodontal ligaments dampen them, and after the action ceases the tooth returns to its initial position, a phenomenon that is not encountered in the case of dental implants (18). Starting from this information presented very briefly, we aimed to present in this material a series of general or particular aspects, which intervene in the design and manufacture of fixed implant-prosthetic restorations, regardless of location: maxillary and/or mandibular.

\section{MATERIAL AND METHOD}

The occlusion of implant-supported restorations has characteristics similar to that of natural teeth and teeth restored with fixed dental prosthesis, so it should be constructed so that it fits into the usual occlusion performed by natural teeth, that is, it should not be built purely and simply a "mechanical gear". The occlusion obtained after creating prosthetic restoration on osseointegrated implants must be created so that all the characteristics of the support mechanism are fulfilled - and when we say this we refer to the avoidance of mechanical overloading of the dental implant, of its connecting components and of the prosthetic superstructure (1-8).
The dentist has the responsibility to choose the optimum place of insertion of the dental implant, so that no paraxial forces are exerted on it and also to diminish the torsion effect that results during the functional movements, the crown acting as a lever towards the distal or towards the vestibular (1-8).

This concept is usually dependent on the anatomical situation. Normally, in the case of partial edentation, the occlusal pattern should be adapted to the existing situation, and the forces that appear at the level of the dental implants should be dispersed so that they are transferred to the bone evenly. One way to accomplish this concept is to use the canine guidance (1-8). The reduction of the forces that are exerted at the level of the dental implants can be obtained also by modeling of occlusal surfaces, which have cusps with rounded angles (smooth cusp slopes) (1-8).

In order to minimize the forces that appear at the level of dental implants, the use of polymeric materials for the occlusal surfaces of artificial teeth is very little justified, given that studies have concluded that, in the case of implant-prosthetic restorations, ceramic materials have much better results, the occlusal surface of the prosthetic restoration is not affected by the abrasion phenomenon (1-8).

Generally, the objectives of obtaining a correct occlusion are the following (1-8):

- maximizing the occlusal function (multiple, stable, simultaneous contacts);

- reducing the negative effects that may occur on antagonistic and adjacent teeth;

- minimizing the abrasion that can occur on the occlusal surfaces;

- reducing fracture risk of dental implant, its connecting components or its superstructure (by eliminating the torsions);

- maintaining the implant's osseointegration (protecting the implant-bone interface) (monitoring, hygiene).

The occlusal features specific to implant-prosthetic treatment, according to Hobkirk, are the following (1-8):

- The insertion place - the possibility of inserting dental implants where the dentist considers that it is the ideal place both from the point of view of the occlusion and from the point of view of the available bone;

- The displacement - an osseointegrated dental implant moves very little under the effect of the forces that appear during functional movements and has a relatively low elasticity;

- The immobility - dental implants cannot be moved by orthodontic forces; 
- The proprioception - the response of proprioceptors from bone level is low;

- The transmission of forces - excessive force may appear in a patient with an extended dental bridge;

- The overloading - the transmission of excessive forces is the key factor, in case of dental implants loss;

- The mechanical connections - almost all dental implants have mechanical connections and their overload leads to failure and loss of dental implant.

Directing forces is mainly done through the following design mechanisms (1-8):

- Type of occlusion and occlusal pattern;

- The superstructure project.

Finally, it became obvious that, when excessive forces appear at the superstructure, the fracture of the osseointegrated interface can occur. The results obtained have increased the interest in the harmful effects that can occur in the case of excessive forces, especially since the meta-analysis has shown that, as soon as osseointegration was achieved, the main cause of the failure of the dental implant and its superstructure it is of a mechanical nature, therefore, prosthetic (1-8).

In the case of dental implants insertion in the lateral areas of the oral cavity, which are treated with dental bridges, during the closing-opening movements, these will be subject to forces with vestibular-oral direction. Thus, implant supported total prosthetic restoration to the mandible is considered contraindicated, due to the flexibility of the bone, which is manifested even in the presence of the restoration, and thus can occur negative effects as (1-8):

- bone resorption around dental implants;

- decementing of the implant-prosthetic superstructure;

- cracking or fracturing of facets and/or even dental implants;

- discomfort feeling for the patient.

To diminish these effects, when bi-terminal edentation is treated through the dental implants, the following measures can be taken (1-8):

- The use of alloys with elastic properties in the construction of the superstructures;

- Reduction of the vestibular-oral size of the bridge, in order to increase the elasticity of the prosthetic work;

- Insertion of additional implants, allowing the use of two or more independent prosthetic works; this variant confers the advantage of increasing the surface area of implants and the degree of retention for the retaining elements.

Starting from these notions regarding the design and manufacture of fixed implant-prosthetic restorations, we developed a 9-point questionnaire, in order to familiarize some dentists involved in the implant-prosthetic rehabilitation therapy (especially those who perform the prosthetic component of this type of implant treatment) (9-21). This questionnaire was then applied to a number of 31 dentists in Bucharest, professionals familiar with the modern techniques of implant-prosthetic rehabilitation of single or multiple teeth edentations. The study took place between September and December 2019. The gender distribution of the dental practitioners included in the study was as follows: 12 subjects were female $(38.71 \%)$, while 19 subjects were male (61.29\%) (Fig. 1). All the subjects involved in the study were between 29 and 66 years old. Next, we will present the questionnaire that was applied to the 31 subjects.

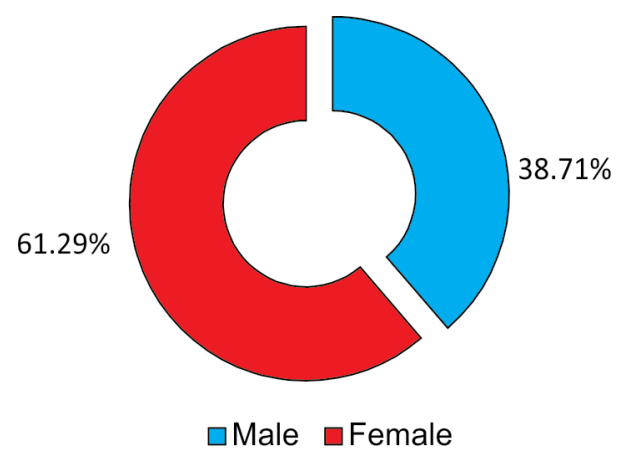

FIGURE 1. Gender distribution of study participants

Questionnaire

1. In the case of dental implants insertion, the dentist has the responsibility to choose the optimal place of insertion of the dental implant because: $\boldsymbol{a}$. No paraxial forces should be exerted on the dental implant. $\boldsymbol{b}$. The torsion effect resulting during the functional movements should be diminished, the crown acting as a distal or vestibular lever; $c$. The future occlusion of prosthetic structures should present a degree of over-occlusion, for the protection of temporomandibular joint (TMJ) structures.

\section{Correct answers: $\boldsymbol{a}, \boldsymbol{b}$.}

2. In order to minimize the forces that appear at the level of the dental implants, for the occlusal surfaces of the artificial teeth of the implant-supported restorations, it would be preferable to use the following materials: $\boldsymbol{a}$. Polymeric materials; $\boldsymbol{b}$. Only stainless dental alloys; c. Dental ceramics, regardless of the support infrastructure; $\boldsymbol{d}$. Copper base alloys. Correct answer: $a$. 
3. The objectives of obtaining a correct occlusion in the implant-prosthetic rehabilitation consist of: a. Maximizing the occlusal function; $\boldsymbol{b}$. Reducing the negative effects that can occur on the antagonistic and adjacent teeth; $c$. Minimizing the abrasion that may occur on the occlusal surfaces; $d$. Reducing the risk of fracture of the dental implant, its connecting components or its superstructure; $\boldsymbol{e}$. Maintaining the osseointegration of the dental implant; $\boldsymbol{f}$. Compulsory equalization of face levels. Correct answers: $a, b, c, d, e$.

4. The occlusal characteristics specific to the implant-prosthetic treatment, according to Hobkirk, depend on the following aspects: a. Insertion place; b. Displacement; c. Immobility; d. Proprioception; $\boldsymbol{e}$. Forces transmission; $\boldsymbol{f}$. Overloading; $\boldsymbol{g}$. Mechanic bonds. Correct answers: $\boldsymbol{a}, \boldsymbol{b}, \boldsymbol{c}, \boldsymbol{d}, \boldsymbol{e}, \boldsymbol{f}, \boldsymbol{g}$.

5. Directing forces in the implant-prosthetic rehabilitation is mainly done through the following design mechanisms: $\boldsymbol{a}$. The insertion place of the dental implant; b. Type of occlusion and occlusal pattern; $\boldsymbol{c}$. The superstructure project; $\boldsymbol{d}$. Physical and mathematical models. Correct answers: $\boldsymbol{a}, \boldsymbol{b}, \boldsymbol{c}$.

6. The meta-analysis has shown that, as soon as osseointegration has been achieved, the main cause of failure of dental implant and its superstructure is: a. Chemical (depends on the material from which the implant-supported restoration is made of); $\boldsymbol{b}$. Mechanical, therefore prosthetic; $\boldsymbol{c}$. Depending on the imaging exams that have been performed. Correct answer: $\boldsymbol{b}$.

7. In the case of insertion of dental implants in the posterior areas of the oral cavity that are treated with dental bridges, during the opening-closing movements of the mouth, they will be subjected to: a. Occlusal loads with a vestibular and oral direction; b. Occlusal loads with a mesial and distal direction; $c$. Occlusal loads with a central and lateral direction. Correct answer: $a$.

8. Following the insertion of a total mandibular prosthetic restoration on dental implants, the following negative effects may occur: $\boldsymbol{a}$. Bone resorption around dental implants; $\boldsymbol{b}$. Decementation of the implant-prosthetic superstructure; $c$. Cracking or fracturing of facets or even dental implants; $d$. Discomfort feeling for the patient; $\boldsymbol{e}$. Loss of natural teeth. Correct answers: $\boldsymbol{a}, \boldsymbol{b}, \boldsymbol{c}, \boldsymbol{d}$.

9. In order to mitigate the negative effects that appear when bi-terminal edentations are rehabilitated with implant-prosthetic restorations, the following measures can be taken: $\boldsymbol{a}$. Use of dental alloys with elastic properties for the superstructures; b. Reduction of the vestibular-oral size of the bridge, in order to increase the elasticity of the prosthetic restoration; $\boldsymbol{c}$. The insertion of additional dental implants, which allows the insertion of two or more independent prosthetic works. Correct answers: $a, b, c$.

\section{RESULTS}

Carefully analyzing the results, we can conclude some important aspects.

To the first question related to the responsibility of the dentist in choosing the optimal place for inserting dental implant, the majority of the respondents (29 representing 93.55\%) answered correctly, namely the lack of paraxial forces and diminishing of torsion effect. Only 2 respondents (representing $6.45 \%$ ) checked all the answer variants (Fig. 2).

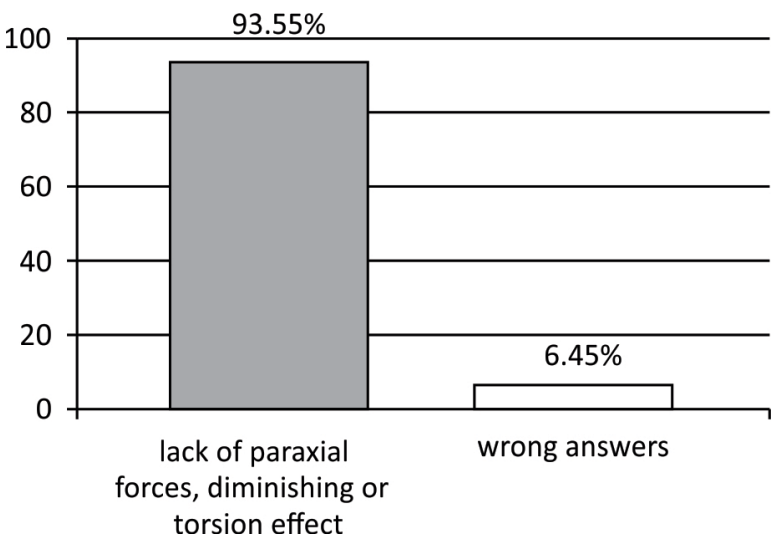

FIGURE 2. Choosing the optimal place for inserting dental implant

Regarding the materials recommended for the occlusal surfaces in order to reduce the forces that occurs at the level of dental implants 26 practitioners (representing $83.87 \%$ ) correctly answered the variant a. Only 5 practitioners (representing 16.13\%) were wrong including beside the correct answer and the stainless dental alloys variant (Fig. 3).

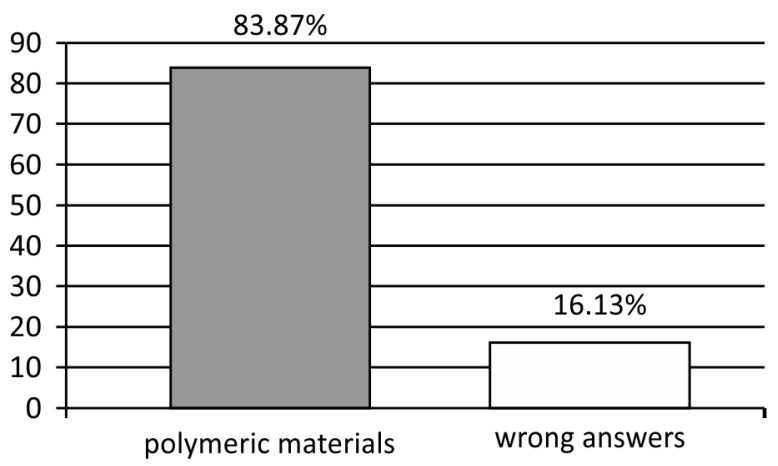

FIGURE 3. Materials recommended for the occlusal surfaces

Concerning the objectives of obtaining a correct occlusion, only 3 participants in the study (repre- 
senting 9.68\%) answered incorrectly (including the variant that required compulsory equalization of face levels). The remaining 28 participants answered correctly (Fig. 4).

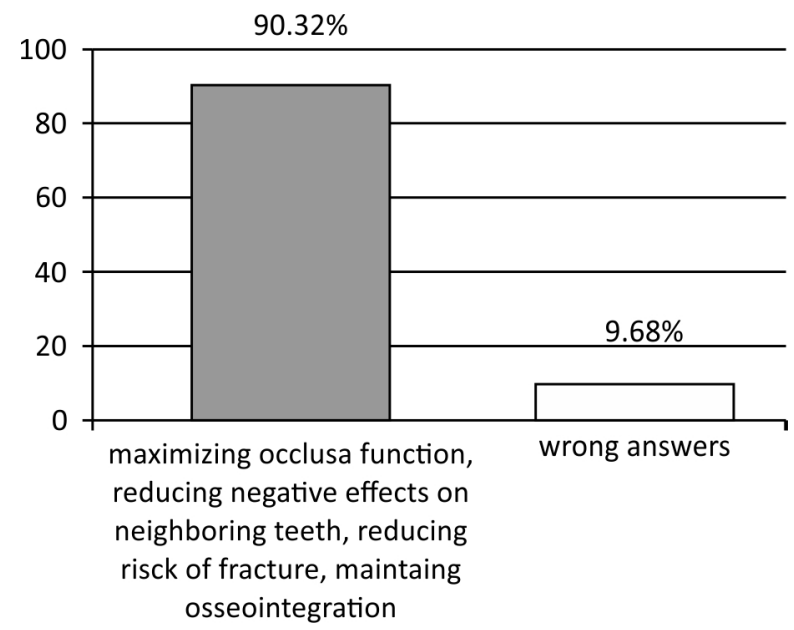

FIGURE 4. Knowing the objectives of obtaining a correct occlusion

It is worth noting that all the doctors involved in the study correctly answered the questions related to the occlusal characteristics and the orientation of the forces in the implant-prosthetic treatment.

Most of the subjects included in the study $(80.65 \%)$ answered correctly regarding the main cause of the failure of the dental implant after osseointegration, namely the mechanical loadings (through the prosthetic elements) (Fig. 5).

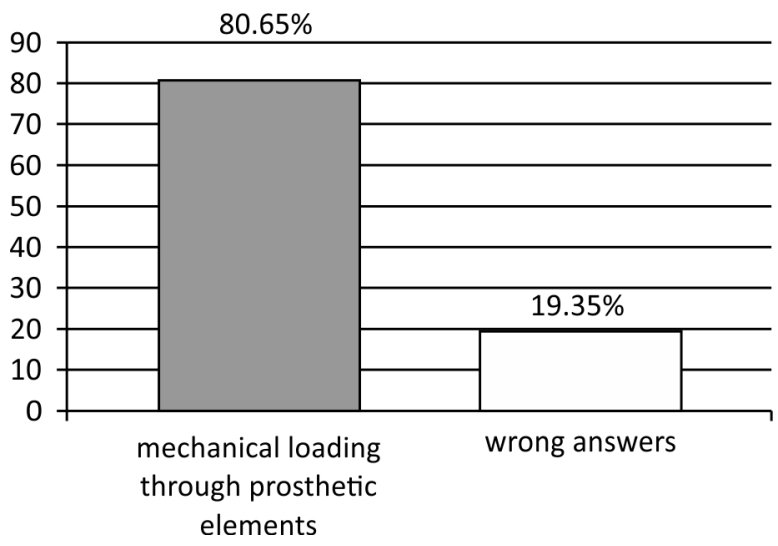

FIGURE 5. Understanding failure causes of the dental implant after osseointegration

For the seventh question related to the pressures to which the implants from the lateral area are subjected in the vertical movements of the mandible, approximately half of the respondents (16 representing $51.61 \%$ ) correctly answered vestibular and oral loadings while the remaining 15 respondents (representing 48.39\%) they answered incorrectly, namely the mesial and distal loadings (Fig. 6).

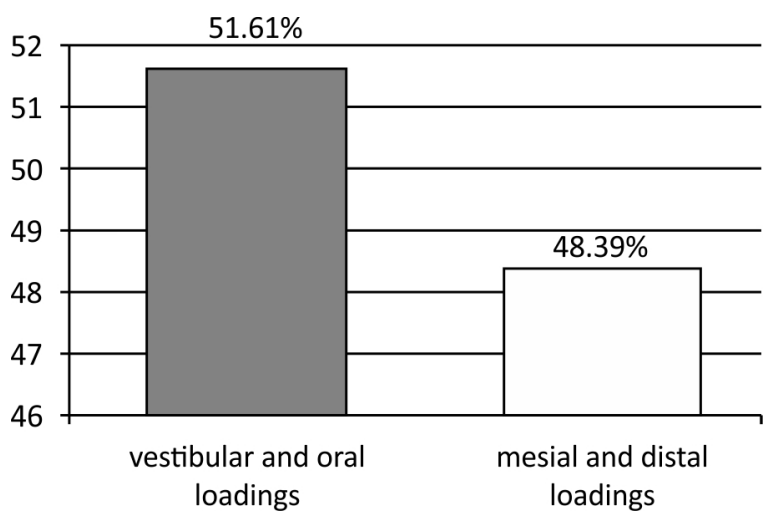

FIGURE 6. Appraisal of the loading on the implants in the lateral area in vertical movements of the mandible.

Most of the practitioners included in the study responded correctly with regard to the negative effects that may occur on total mandibular prosthetic restorations on implants, and only 1 practitioner omitted a correct answer (Fig. 7).

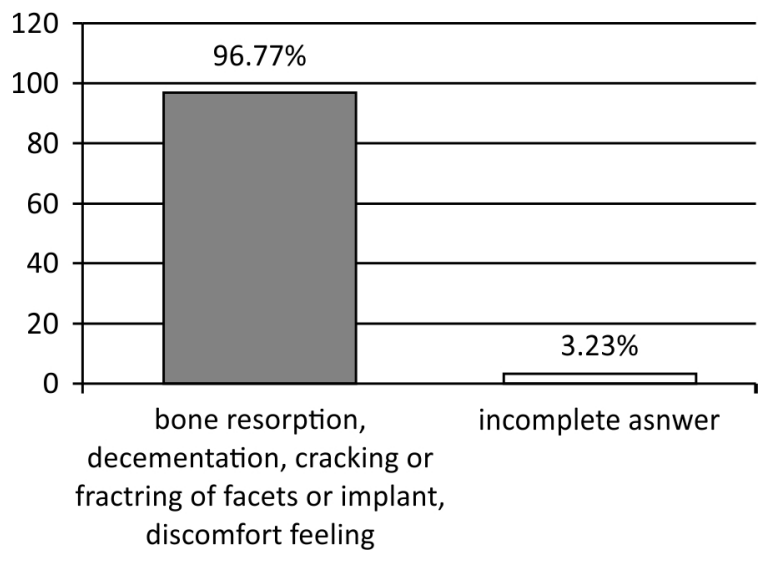

FIGURE 7. The negative effects that can occur after the application of the mandibular total prosthetic restorations

At the last question, we note that all the doctors included in the study have correctly answered about the measures to be taken to mitigate the negative effects that appear in bi-terminal edentation restored with implant-prosthetic restorations.

\section{DISCUSSIONS}

Implantology, similar to dentistry, is a complex science and each stage of the work flow is strongly linked to the other stages. Thus, the design of the treatment plan must make connections between the insertion, osseointegration, loading of the implant as well as its subsequent use.

In all branches of dental medicine, it is necessary for dentists to analyze and evaluate the dental contacts that are made between the teeth or between prosthetic restorations, whether we discuss classi- 
cal or implant-supported prosthetic restorations, fixed or mobile. When evaluating dental occlusion, dental practitioners must consider the presence of simultaneous dental contacts, the moment of articulation and the forces that appear, when maximum inter-cuspidation is performed.

From 1993 to the present, the long-term studies on the life of the single implants have confirmed that this treatment method is the most appropriate, when a limited edentulous space created by the absence of a single tooth, regardless its localization. In the specialty literature, there are more studies on single implants than on any other treatment method, and all the existing studies have shown that single implants have the highest success rate.

The occlusal forces that appear at the level of an implant-supported prosthetic restoration are transmitted through abutments to the dental implants and bone. If there is no proper occlusal balance and the parafunctions are not removed, failure of dental implant treatment is only a matter of time. As Gross in 2008 in a literature review about "Occlusion in implant dentistry" has high lightened that parafunctions can create destructive loads that negatively affect implant, surrounding bone, and prosthetic restorations (22). From this point of view, the insertion of the implant should take into account not only the bone supply, but also the use of the future prosthetic work.

The materials from which the prosthetic works are made must be chosen not to affect the bone support in which the implant is inserted. Both the materials from which the prosthetic work is made and the materials used to fix it to the dental implants, play a crucial role in reducing the forces at the occlusal level so that at the bone level their value is as low as possible to avoid bone resorption and subsequent implant loss. Studying the correlation between restorative materials from occlusal surfaces and force distribution for implant-supported prostheses, Inan et al. concluded that ceramic materials causes a great stress distribution around the apex of the implant, while minimum stress distribution is observed for composite materials (23).

In the case of implant-prosthetic restorations, the use of distal extensions with one or two dental units is frequently encountered, apparently without controversy on this subject. In contrast, performing distal extensions on natural teeth can result in increased failure rates for fixed prosthetic works. In any case, more incidents were reported due to technical errors in the case of implant-prosthetic restorations provided with distal extension than in the case of those who did not have such restorations.
Based on the success of the implant-prosthetic restorations and the long-term results published in the specialized literature, it can be concluded that a varied number of methods for recording the mandibular movement and performing the occlusal morphology are acceptable for implant-prosthetic rehabilitation, as they are for prosthetic restorations performed on natural teeth.

A question still remains, whether the occlusion of prosthetic restorations on natural teeth should differ or not from that of implant restorations. At present, it seems prudent to accept the principles and methods applied in fixed dentistry performed on natural teeth also in the case of implant-prosthetic restorations.

Equally important is the occlusal balance after the implant-prosthetic restoration is made, any paraxial force will have the effect of bone resorption and destabilization of the dental implant. The occlusal principles that have been established for restorations on natural teeth cannot be fully applied when it comes to implant supported restorations. There is a major difference between the ways the teeth are fixed in the bone, through the periodontal ligaments, while the dental implant has a much stiffer fixation, occlusal forces being transmitted much more directly to the bone support. Kim el al., in a study regarding "Occlusal considerations in implant therapy", suggested that there are three main factors to be considered in the implant occlusion: increase of support area, improve force direction, and reduce force magnification (24). Failure to comply with this guide lines may cause the failure for osseointegrated implants, on medium- or long-term due to occlusal overload (25).

All these aspects related to the long-term success of prosthetic restorations on implants are increasingly outlined at a theoretical level, practical courses, as well as scientific meetings with the role of disseminating new information obtained through hundreds of studies in the field. But, more importantly, practitioners should know and apply this new information as we have shown in our study.

\section{CONCLUSIONS}

Most of the dental practitioners involved in this study (over $80 \%$ ) have proven solid knowledge regarding the design and manufacture of implant-prosthetic restorations, which denotes a very good training both theoretically and practically.

The survival rate of dental implants depends not only on the insertion surgical technique and their osseointegration, but also on the correctness of the implant-supported prosthetic restoration. 
It is very important that the prosthetic loading of dental implants is performed correctly from the point of view of the dental occlusion, and the forces that are exerted on the whole dental crown-dental implant assembly do not lead to the loss of the obtained osseointegration.

\section{REFERENCES}

1. Andrei CA., Cotruță Jimenez-Pulido AM, Marcov EC et al. Tendințe moderne în științele biomedicale, Vol. II. Editura Matrix, București, 2019.

2. Hobkirk JA. Occlusion and implant restoration. In: Klineberg I. and Jagger R. (eds.). Occlusion and clinical practice: An evidence-based approach. Edinburgh: Wright/Elsevier; 2004;(13)119.

3. Augustin M, Carabela M, Olteanu I et al. Implanturile endoosoase osteointegrate în stomatologie. Ed. Sylvi, 1995; pp. 19-21, 23, 45, 49-51.

4. Ioniţă S, Petre A. Ocluzia dentară. Ed. Didactică şi Pedagogică, Bucureşti. 2006; pp. 135-139.

5. Bratu D, Ferzer W, Bratu E et al. Puntea pe implante. Ed. Helicon, Timișoara, 1996, p. 101.

6. Misch CE. Contemporary implant dentistry. 3rd Ed., Mosby Elsevier, St. Louis, 2008.

7. Bratu D, Nussbaum R. Bazele clinice şi tehnice ale protezării fixe. Ediţia a III-a. Ed. Medicală, București, 2011.

8. David D. Morfologie dentară. Carte de curs. Ed. Carol Davila, Bucureşti. 2006, pp. 110-111.

9. Bodnar DC, Dimova C, Cristache CM et al. Dental management of patient with psychiatric disorders. Modern Medicine. 2010;17(10):538543.

10. Popescu FD, Popescu SM, Burlibașa M. Platinum group metals as contact allergens in oral rehabilitation. Metalurgia International. 2010;15:12-18

11. Ionescu CA., Popovici LR, Mocuța $D$ et al. The quality of human life from the perspective of sustainable development. Metalurgia International. 2009;14:41-43.

12. Eftimie-Totu E, Cristache CM, Isildak $S$ et al. Preliminary studies on citotoxicity and genotoxicity assesment of the PMMA - TiO2 nanocomposites for stereolithographic complete dentures manufacturing. Revista de Chimie. 2018;69(5):1160-1165.

13. Mocuța $D$, Popovici IA, Burlibașa $L$ et al. Impact of the living conditions on population health. Metalurgia International. 2009;14:17-19.

\section{Acknowledgement}

In this article, all authors have equal contribution with the first author.

Conflict of interest: none declared Financial support: none declared

14. Jamous $\mathrm{S}$, Burlibașa L. New insights in molecular analysis of gene regulation - an epigenetic overview. Letters in Applied Nanobioscience. 2018;7(1-2):511-522.

15. Burlibașa M, Tănase $G$, Muntianu L et al. Quality of life, a multidisciplinary concept with economic and social impacts in medical practice. Metalurgia International. 2010;15(4):88-90.

16. Ispas DC, Eftene OA, Burlibașa M et al. Implications of titanium in orthodontics and dental facial orthopedics. Metalurgia International. 2011;16(10):72-74.

17. Burlibasa $L$, Domnariu C. Epigenetic landscape of human diseases. Acta Medica Transilvanica. 2018;23(2):33-37.

18. Bodnar DC, Burlibașa L, Vârlan C et al. Mercury, biocompatibility and its impact on environment. Metalurgia International. 2009;14:95-100.

19. Cristache CM, Burlibașa M, Cristache $\mathrm{G}$ et al. Zirconia and its biomedical applications. Metalurgia International. 2011;16(7):18-23.

20. Mihai $A$, Chifiriuc CM, Lazăr V et al. Microbial biofilms in dental medicine in reference to implanto-prosthetic rehabilitation. Rev Chir Oro-maxilo-fac Implantol. 2008;1(1):9-13.

21. Sfarghiu LG, Oancea L, Lițescu R et al. The influence of disinfectants incorporation on die stone linear expansion. Romanian Biotechnological Letters. 2016;21(3):11585-90.

22. Gross MD. Occlusion in implant dentistry. A review of the literature of prosthetic determinants and current concepts. Aust Dent J. 2008;53 Suppl 1:S60-S68.

23. Inan O, Kesim B. Evaluation of the effects of restorative materials used for occlusal surfaces of implant-supported prostheses on force distribution. Implant Dent. 1999;8(3):311-316.

24. Kim Y, Oh TJ, Misch CE, Wang HL. Occlusal considerations in implant therapy: Clinical guidelines with biomechanical rationale. Clin Oral Implants Res. 2005;16(1):26-35.

25. Rilo B, da Silva JL, Mora MJ, Santana U. Guidelines for occlusion strategy in implant-borne prostheses. A review. Int Dent J. 2008;58(3):139-145. 